\section{Quality of life of patients with cancer undergoing chemotherapy in hospitals in Belo Horizonte, Minas Gerais State, Brazil: does individual characteristics matter?}

\author{
Qualidade de vida de pacientes com câncer \\ submetidos a quimioterapia em hospitais \\ em Belo Horizonte, Minas Gerais, Brasil: \\ as características individuais importam?
}

\section{Calidad de vida de pacientes con cáncer sometidos a quimioterapia en hospitales de Belo Horizonte, Minas Gerais, Brasil: ¿importan las características individuales?}

Daniela Pena Moreira 1

Giovana Paula Rezende Simino 1

Ilka Afonso Reis 1

Marcos Antonio da Cunha Santos 1

Mariangela Leal Cherchiglia 1

\begin{abstract}
This study aims to evaluate changes in quality of life of cancer patients at the beginning of the first and the second cycle of chemotherapy (CT) in hospitals in Belo Horizonte, Minas Gerais State, Brazil. Longitudinal, prospective, descriptive study with a quantitative approach. We enrolled 230 patients, from a broader cohort, diagnosed with the five most frequent types of cancer (breast, colorectal, cervical, lung, and head and neck), aged 18 years or older, who were initiating CT for the first time. quality of life was assessed with the EORTC QLQ-C30 version 3, applied at the beginning of the first and second CT cycle. The paired Wilcoxon test was used to identify differences in quality of life between the two time points. A multivariate linear regression analysis was performed using the bootstrap method to investigate potential predictors of global health Status/quality of life. There was a significant increase in patients' emotional function scores $(p<0.001)$ as well as symptom scores for pain $(p=0.026)$, diarrhea $(p=0.018)$, and nausea/vomiting $(p<$ 0.001 ) after initiation of CT. Widowhood was associated with improvements in the global health Status/quality of life $(p=0.028)$, whereas the presence of cervical cancer $(p=0.034)$ and being underweight $(p=0.033)$ were related to poorer global health status/quality of life scores. CT has detrimental effects on patients' physical health but, on the other hand, it leads to improvements in the emotional domain. Patients' individual characteristics at the beginning of CT are associated with changes in their quality of life. Our study could help identify these characteristics.
\end{abstract}

Neoplasms; Quality of Life; Drug Therapy; Health Profile; Cytotoxins

\author{
Correspondence \\ D. P. Moreira \\ Rua Bernardo Guimarães 341, apto. 102, Belo Horizonte, MG \\ 30140-080, Brasil. \\ danipenamoreira@gmail.com \\ 1 Universidade Federal de Minas Gerais, Belo Horizonte, Brasil.
}




\section{Background}

Cancer is a public health problem that affects individuals of all ages and genders. In the different regions of the world, a high diversity in the profile of the disease can be found, as well as varying incidence and mortality rates. These differences are related to lifestyle, economic development, human development index (HDI), sociodemographic characteristics, and unequal access to healthcare 1.

According to the Brazilian National Cancer Institute (INCA), 625,000 new cancer cases are expected for the 2020-2022 triennium in Brazil. Except for non-melanoma skin cancer, prostate cancer is the most common type in men, with 65,840 cases - 1,270 in Belo Horizonte, a city in the State of Minas Gerais, Brazil - and breast cancer is the most common type in women, with 66,280 cases $-1,800$ in Belo Horizonte 2 . In addition to high incidence and prevalence, malignant tumors are the second leading cause of death in Brazil and, in some countries, they are already the first cause of death in individuals younger than 70 years 1 .

Multiple treatments for cancer are available and the most common are surgery, radiation therapy, and chemotherapy (CT). The course of these treatments involves physical and emotional stress, as well as changes in the patients' cognitive capacity 3,4, which can ultimately affect their quality of life. According to the World Health Organization (WHO), quality of life is "an individual's perception of their position in life in the context of the culture and value systems in which they live and in relation to their goals, expectations, standards, and concerns" 5 (p. 1405). Quality of life is a multidimensional concept and should be assessed only by the subjects themselves. In the field of health, quality of life assessment instruments are increasingly used and can be self-administered or carried out by interviews 6 .

International regulatory agencies interest in the experience of cancer patients are increasing, and quality of life is a very relevant measure for decision making 7 . In clinical practice, outcomes reported by patients can increase the perception of symptoms by healthcare professionals, enhance the efficiency of consultations, and decrease communication barriers, increasing discussions and improving patients' quality of life 8 . Studies have shown that the use of quality of life indicators improves communication between physicians and patients, shared decision making 9,10,11, and promotion of health 12 . Despite the different characteristics that each type of cancer carries, CT is a systemic treatment with multiple adverse effects that are common among patients, such as myelosuppression, nausea, vomiting, and fatigue 13. These effects can potentially lead to relevant effects on patient quality of life, in addition to interruptions and delays in treatments. Healthcare professionals look after different types of cancer patients, emphasizing the individual characteristics of these patients that can be predictors of quality of life. Therefore, this study aims to assess the quality of life of cancer patients with different types of cancer undergoing CT in Belo Horizonte.

\section{Methods}

\section{Study population and design}

A longitudinal, prospective, descriptive study with a quantitative approach. The study is part of a cohort 14 observing the incidence of nausea and vomiting in chemotherapy-naive patients, as well as the quality of life using the European Organization for Research and Treatment of Cancer Quality of Life Questionnaire Core 30(EORTC QLQ-C30). The original study enrolled patients aged 18 or older, who were initiating outpatient chemotherapy treatment for the first time. Exclusion criteria were: nausea or vomiting in the 24 hours prior to the first day of data collection, abdominal radiation therapy concomitantly to chemotherapy, sleepiness, and inability (physical or mental) to communicate. Patients were recruited at three reference cancer hospitals in the city of Belo Horizonte. The Institutional Review Board of the Federal University of Minas Gerais (COEP/UFMG) approved the study. We obtained approval from the participating hospitals and all patients signed an informed consent form before starting data collection. 


\section{Data collection}

Sociodemographic data were collected using an instrument developed by the investigators, the Clinical and Sociodemographic Identification Questionnaire (IC), which was administered to subjects on the first day of the first chemotherapy cycle. Clinical data were collected from patient charts. The EORTC QLQ-C30 version 3.0 was at two different timepoints - on the first day of the first chemotherapy cycle (timepoint 1) and on the first day of the second chemotherapy cycle (timepoint 2) - in order to assess the quality of life of patients. The EORTC QLQ-C30 version 3.0 instrument is composed of 30 questions and divided into multiple-item and single-item scales. In total, five functional scales (physical, role, emotional, cognitive and social), nine symptoms/single-item scales (fatigue, nausea/vomiting and pain, dyspnea, insomnia, appetite loss, constipation, diarrhea and financial difficulties) and a global health status/quality of life scale were included. Scales are linearly transformed into scores ranging from 0 to 100 . High scores in the global health status/quality of life and functional scales represent high quality of life and a healthy functional level. In symptoms or single-item scales, high scores represent a high level of symptoms or problems 15. Data were collected from June to November 2015.

\section{Statistical analyses}

The descriptive analysis was based on the calculation of absolute and relative frequencies for categorical variables, and measures of central tendency and dispersion (mean, median, standard deviation, and minimum and maximum) for quantitative variables. For each scale of the instrument, patient scores were calculated according to the instructions in the manual 15. The distribution of scores was not considered normal (Shapiro-Wilk test). The paired Wilcoxon test was used to identify significant differences between EORTC QLQ-C30 timepoint 1 and timepoint 2. A regression model was constructed with GHS/QoL at timepoint 2 as the dependent variable, adjusted by global health status/quality of life timepoint 1 , the treating hospital and the stage of cancer at the time of diagnosis. Sociodemographic, clinical, and treatment-related variables were used as the independent ones. For the variable selection, univariate models with global health status/quality of life at timepoint 2 were considered as dependent variable and clinical, sociodemographic or treatment-related as the independent variable. A p-value $<0.2$ was used as criteria for this initial selection. Based on this set of pre-selected variables, a final multiple linear regression model was considered based on the backward selection technique. This method initially considers all variables in the model. Adjustments were made by removing variables one by one, and those that did not meet a certain significance level are excluded. This procedure was repeated until all variables were significant at $5 \%$ level. The non-parametric bootstrap method was used to calculate confidence intervals and p-values related to the regression model, with 2,000 replications. Also, 95\% confidence intervals and a 0.05 level of significance were used. All analyses were performed with R statistical software (http://www.r-project.org).

\section{Compliance with ethical standards}

All procedures performed in studies involving human participants were in accordance with the ethical standards of the institutional and/or national research committee and with the 1964 Helsinki Declaration and its later amendments or comparable ethical standards.

The Institutional Review Board at the UFMG, approved the study (CAAE: 36059514.5.0000.5149) and informed consent was obtained from all individual participants included in the study. This article does not contain any studies with animals performed by any of the authors. 


\section{Results}

\section{The patients}

We selected the five most frequent types of cancer in the original cohort (breast, colon and rectum, cervix, head and neck, and lung), which resulted in 230 patients (65.3\% of the 315 patients). This choice would make possible perform the statistical analysis in a proper way (Figure 1).

\section{Figure 1}

Inclusion flow and follow-up of patients. Belo Horizonte, Minas Gerais State, Brazil, 2020.

Other types of cancer $(n=85)$ :

- Esophageal $(n=14)$

- Ovarian $(n=13)$

- Stomach $(n=9)$

- Diffuse non-Hodgkin's lymphoma $(n=8)$

- Prostate $(n=8)$

- Non-specific $(n=7)$

- Others (11 types) $(n=26)$
549 patients started CT

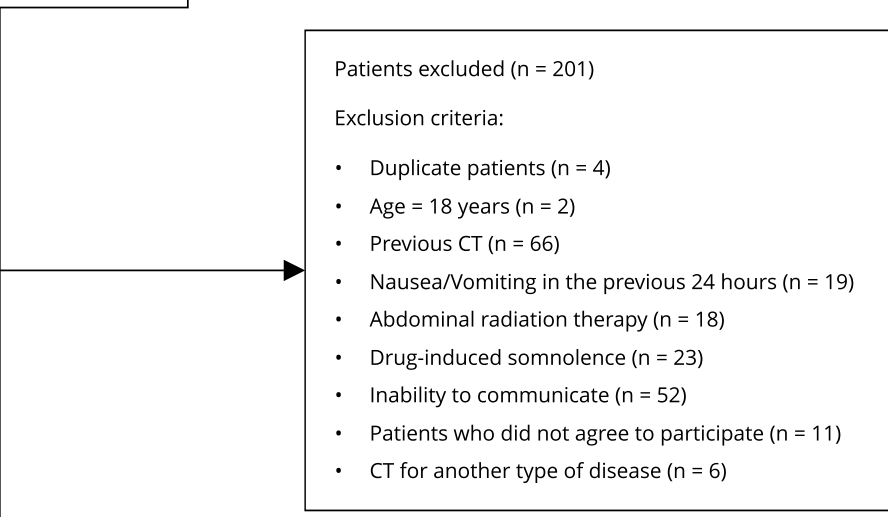

348 patients met the original cohort criteria

Loss of follow-up or EORTC QLQ-C30 incomplete $(n=33)$

- $\quad$ CT interruption $(n=3)$

- Death $(n=1)$

- Questionnaire EORTC QLQ-C30 3.0 incomplete $(n=7)$

- Loss of follow-up due to suspension or change of treatment $(n=16)$

- $\quad C T$ during hospitalization $(n=6)$ 
Demographic data, clinical characteristics and variables related to the treatment of 230 patients according to the type of cancer are described in Table 1. Most study subjects were female, with a mean age of 56.1 years, self-declared mixed-race, and married. The mean time of schooling was 6.2 years, $14.3 \%$ had no income, and most (45.2\%) did not earn more than one minimum wage (USD 200) - considering the Brazilian minimum wage in 2015 and the USD to Brazilian real exchange rate in December 2015. Regarding the lifestyle of study participants, most were or currently are tobacco and alcohol users. As for clinical characteristics, most subjects had breast cancer classified as stages III and IV at the time of diagnosis. Most patients were not overweight and had one comorbidity. The most prevalent comorbidities in this study were systemic hypertension followed by diabetes mellitus, depression, and cardiovascular diseases. According to the type of cancer, two of them are exclusively female (breast and cervix), and the other types, females represented half of the patients with lung cancer, $54.3 \%$ of the colon and rectum and only $15 \%$ of the head and neck. The patients with colon and rectal and lung cancer were older, 60.5 and 59.4 years old, respectively, and for all types of cancer, the most participants were mixed-race and married. Regarding years of study, the lowest averages were found for patients with head and neck and cervix cancer, 5.3 years for both, and patients with lung cancer had the highest proportion of complete high school (21.3\%). The frequency of patients without income was higher among patients with cervical cancer (31.8\%) and the highest proportion of patients who received more than two minimum wages was those with lung cancer (31.3\%). More than half of the patients with head and neck and lung cancer were smokers, $67.5 \%$ and $65.6 \%$, respectively, and $92.5 \%$ of patients with head and neck cancer were alcoholics. In all types of cancer, patients were diagnosed more frequently in stages III and IV and only for breast cancer the majority were obese (44.1\%). Most patients with cervical and head and neck cancer did not have any comorbidity, $40.9 \%$ and $47.5 \%$, respectively.

More than a half of the patients had previously undergone a different type of treatment, and the mean time between diagnosis and the first treatment was 50.6 days. The most frequent chemotherapy protocol in this study was cisplatin (CDDP). The mean distance traveled by subjects for chemotherapy treatments was 95.9 kilometers $(\mathrm{km})$ and $34.3 \%$ were residents of Belo Horizonte. Although $20.9 \%$ of the sample stated to have private health insurance, most chemotherapy treatments were covered by the Brazilian Unified National Health System (SUS). Most patients with cervix, head and neck and lung cancer underwent treatment prior to CT, $61.4 \%, 62.5 \%$ and $71.9 \%$, respectively. Patients with cervical cancer presented the highest average number of days to start treatment (63.7 days) and 50\% of these patients started treatment after 60 days. Furthermore, they traveled long distances from the municipality they dwell to the treatment municipality, with an average of $117 \mathrm{~km}$. Lung cancer patients presented the longest distance traveled, with an average of $119 \mathrm{~km}$.

\section{Quality of life}

The Wilcoxon paired test identified virtually no changes in the global health status/quality of life score of patients between timepoints 1 and 2, and the scores were considered high. In the median score for emotional function a significant improvement at timepoint $2(\mathrm{p}<0.001)$ was identified. Regarding symptoms, a significant increase in the median scores for nausea and vomiting $(\mathrm{p}<0.001)$ and diarrhea $(\mathrm{p}=0.018)$ was detected, and a significant drop in the median scores for pain $(\mathrm{p}=0.026)$ at timepoint 2. No significant difference between the timepoints on other scales (Table 2) was observed.

When looking at the association between clinical and sociodemographic variables, we observed that marital status, type of cancer, and BMI were predictors of changes in global health status/quality of life. After the initiation of treatment, patients with cervical cancer reported deterioration of their global health status/quality of life when compared to those with breast cancer $(\beta=-7.97, p=0.034)$ as well as underweight patients compared with those in their normal weight range $(\beta=-9.03, \mathrm{p}=$ $0.033)$. Widowed subjects reported improvement of their global health status/quality of life score at timepoint 2 when compared to married subjects $(\beta=6.9, \mathrm{p}=0.028)$ (Table 3 ). 
Table 1

Distribution of cancer patients undergoing chemotherapy (CT) by sociodemographic, clinical, and treatment-related variables.

\begin{tabular}{|c|c|c|c|c|c|c|}
\hline & $\begin{array}{c}\text { All } \\
(n=230)\end{array}$ & $\begin{array}{l}\text { Breast } \\
(n=59)\end{array}$ & $\begin{array}{l}\text { Colon and } \\
\text { rectum }(n=55)\end{array}$ & $\begin{array}{c}\text { Cancer type } \\
\text { Cervix } \\
(n=44)\end{array}$ & $\begin{array}{l}\text { Head and neck } \\
\qquad(n=40)\end{array}$ & $\begin{array}{l}\text { Lung } \\
(n=32)\end{array}$ \\
\hline \multicolumn{7}{|l|}{$\operatorname{Sex}(\%)$} \\
\hline Female & $156(67.8)$ & $59(100.0)$ & $31(56.4)$ & $44(100.0)$ & $6(15.0)$ & $16(50.0)$ \\
\hline \multicolumn{7}{|l|}{ Age (years) } \\
\hline Mean (SD) & $56(12.9)$ & $53.4(13.4)$ & $60.1(13.4)$ & $50.6(13.5)$ & $58.1(9.4)$ & $59.4(10.7)$ \\
\hline Median (min-max) & $57(22-88)$ & $51(31-86)$ & $60(31-88)$ & $51.5(22-80)$ & $59(41-78)$ & $59.5(26-80)$ \\
\hline \multicolumn{7}{|l|}{ Race/Color (\%) } \\
\hline Mixed-race & $134(58.3)$ & 34 (57.6) & $28(50.9)$ & $26(59.1)$ & $25(62.5)$ & $21(65.6)$ \\
\hline White & $57(24.8)$ & $16(27.1)$ & $16(29.1)$ & $10(22.7)$ & $9(22.5)$ & $6(18.8)$ \\
\hline Black & $27(11.7)$ & $9(15.3)$ & $7(12.7)$ & $7(15.9)$ & $2(5.0)$ & $2(6.2)$ \\
\hline No information & $12(5.2)$ & $0(0.0)$ & $4(7.3)$ & $1.0(2.3)$ & $4(10.0)$ & $3(9.4)$ \\
\hline \multicolumn{7}{|l|}{ Marital status (\%) } \\
\hline Married/Stable union & $120(52.2)$ & $32(54.2)$ & $30(54.5)$ & $18(40.9)$ & $21(52.5)$ & 19 (59.4) \\
\hline Single & $43(18.7)$ & $14(23.7)$ & $10(18.2)$ & $8(18.2)$ & $7(17.5)$ & $4(12.5)$ \\
\hline Divorced/Separated & $41(17.8)$ & $7(11.9)$ & $10(18.2)$ & $9(20.5)$ & $11(27.5)$ & $4(12.5)$ \\
\hline Widow/widower & $26(11.3)$ & $6(10.2)$ & $5(9.1)$ & $9(20.5)$ & $1(2.5)$ & $5(15.6)$ \\
\hline \multicolumn{7}{|l|}{ Schooling (years) } \\
\hline Mean (SD) & $6.2(4.2)$ & $7.0(3.8)$ & $6.0(3.7)$ & $5.3(3.7)$ & $5.3(4.1)$ & $7.1(5.7)$ \\
\hline Median (min-max) & $5(0-17)$ & $7(4-16)$ & $4(0-14)$ & $4(0-12)$ & $4(0-16)$ & $7(0-17)$ \\
\hline Illiterate & $18(7.8)$ & $1(1.7)$ & $3(5.5)$ & $4(9.1)$ & $5(12.5)$ & $5(15.6)$ \\
\hline $1-4$ & $93(40.4)$ & $21(35.6)$ & $25(45.5)$ & $20(45.5)$ & $18(45.0)$ & $9(28.1)$ \\
\hline $5-8$ & $49(21.3)$ & $16(27.1)$ & $11(20.0)$ & $9(20.5)$ & $9(22.5)$ & $4(12.5)$ \\
\hline $9-12$ & $54(23.5)$ & $19(32.2)$ & $14(25.5)$ & $10(22.7)$ & $5(12.5)$ & $6(18.8)$ \\
\hline$>12$ & $12(5.2)$ & $2(3.4)$ & $1(1.8)$ & $0(0.0)$ & $2(5.0)$ & $7(21.9)$ \\
\hline No information & $4(1.74)$ & $0(0.0)$ & $1(1.8)$ & $1(2.3)$ & $1(2.5)$ & $1(3.1)$ \\
\hline \multicolumn{7}{|l|}{ Children (\%) } \\
\hline Yes & $197(85.7)$ & $51(86.4)$ & $42(76.4)$ & $43(97.7)$ & $35(87.5)$ & $26(81.2)$ \\
\hline \multicolumn{7}{|l|}{ Number of children } \\
\hline Mean (SD) & $3.2(2.7)$ & $2.7(2.5)$ & $2.7(2.3)$ & $4.7(3.3)$ & $3.0(2.5)$ & $3.1(2.1)$ \\
\hline \multicolumn{7}{|l|}{ Individual income (USD) } \\
\hline Mean (SD) & $254.6(321.1)$ & $193.7(130.2)$ & $266.6(150.5)$ & $141.5(149.0)$ & $247.8(322.0)$ & $509.9(660.8)$ \\
\hline No income (minnimun wave) & $33(14.3)$ & $7(11.9)$ & $4(7.3)$ & $14(31.8)$ & $6(15.0)$ & $2(6.2)$ \\
\hline Up to 1 & $104(45.2)$ & $36(61.0)$ & $22(40.0)$ & $19(43.2)$ & $18(45.0)$ & $9(28.1)$ \\
\hline $1-2$ & $67(29.1)$ & $14(23.7)$ & $21(38.2)$ & $9(20.5)$ & $12(30.0)$ & $11(34.4)$ \\
\hline$>2$ & $26(11.3)$ & $2(3.4)$ & $8(14.5)$ & $2(4.6)$ & $4(10.0)$ & $10(31.2)$ \\
\hline \multicolumn{7}{|l|}{ Smoking (\%) } \\
\hline Yes. I was a smoker & $100(43.5)$ & $22(37.3)$ & $16(29.1)$ & $14(31.8)$ & $27(67.5)$ & $21(65.6)$ \\
\hline Yes. I am a smoker & $27(11.7)$ & $4(6.8)$ & $4(7.3)$ & $5(11.4)$ & $11(27.5)$ & $3(9.4)$ \\
\hline I have never been a smoker & $103(44.8)$ & $33(55.9)$ & $35(63.6)$ & $25(56.8)$ & $2(5.0)$ & $8(25.0)$ \\
\hline \multicolumn{7}{|l|}{ Alcohol consumption (\%) } \\
\hline Yes. I already drank alcohol & $107(46.5)$ & $19(32.2)$ & $23(41.8)$ & $14(31.8)$ & $37(92.5)$ & $14(43.8)$ \\
\hline Yes. I drink alcohol & $30(13.0)$ & $11(18.6)$ & $9(16.4)$ & $4(9.1)$ & $2(5.0)$ & $4(12.5)$ \\
\hline I never drank alcohol & $93(40.4)$ & $29(49.2)$ & $23(41.8)$ & $26(59.1)$ & $1(2.5)$ & $14(43.8)$ \\
\hline
\end{tabular}

(continues) 


\begin{tabular}{|c|c|c|c|c|c|c|}
\hline & $\begin{array}{c}\text { All } \\
(n=230)\end{array}$ & $\begin{array}{l}\text { Breast } \\
(n=59)\end{array}$ & $\begin{array}{c}\text { Colon and } \\
\text { rectum }(n=55)\end{array}$ & $\begin{array}{c}\text { Cancer type } \\
\text { Cervix } \\
(n=44)\end{array}$ & $\begin{array}{l}\text { Head and neck } \\
\qquad(n=40)\end{array}$ & $\begin{array}{l}\text { Lung } \\
(n=32)\end{array}$ \\
\hline \multicolumn{7}{|l|}{ Stage (\%) } \\
\hline 1 & $11(4.8)$ & $3(5.1)$ & $1(1.8)$ & $5(11.4)$ & $0(0.0)$ & $2(6.2)$ \\
\hline ॥ & $48(20.9)$ & $23(39.0)$ & $6(10.9)$ & $17(38.6)$ & $1(2.5)$ & $1(3.12)$ \\
\hline III & $98(42.6)$ & $30(50.8)$ & $25(45.5)$ & $19(43.2)$ & $13(32.5)$ & $11(34.4)$ \\
\hline IV & 68 (29.6) & $3(5.1)$ & $20(36.4)$ & $3(6.8)$ & $26(65.0)$ & $16(50.0)$ \\
\hline No information & $5(2.2)$ & $0(0.0)$ & $3(5.5)$ & $0(0.0)$ & $0(0.0)$ & $2(6.2)$ \\
\hline \multicolumn{7}{|l|}{ Hospital (\%) } \\
\hline 1 & $51(22.2)$ & 20 (33.9) & $13(23.6)$ & $5(11.4)$ & $4(10.0)$ & $9(28.1)$ \\
\hline$\|$ & $116(50.4)$ & $12(20.3)$ & $28(50.9)$ & $31(70.5)$ & $23(57.5)$ & $22(68.8)$ \\
\hline III & $63(27.4)$ & $27(45.8)$ & $14(25.5)$ & $8(18.2)$ & $13(32.5)$ & $1(3.12)$ \\
\hline \multicolumn{7}{|l|}{ Comorbidities (\%) } \\
\hline None & 78 (33.9) & 20 (33.9) & $15(27.3)$ & $18(40.9)$ & $19(47.5)$ & $6(18.8)$ \\
\hline 1 & 79 (34.3) & $21(35.6)$ & $23(41.8)$ & $12(27.3)$ & $11(27.5)$ & $12(37.5)$ \\
\hline 2 & $47(20.4)$ & $10(16.9)$ & $12(21.8)$ & $9(20.5)$ & $9(22.5)$ & $7(21.9)$ \\
\hline 3 or more & 25 (10.9) & $8(13.6)$ & $4(7.3)$ & $5(11.4)$ & $1(2.5)$ & $7(21.9)$ \\
\hline No information & $1(0.4)$ & $0(0.0)$ & $1(1.8)$ & $0(0.0)$ & $0(0.0)$ & $0(0.0)$ \\
\hline \multicolumn{7}{|l|}{ BMI (\%) } \\
\hline Low weight & $17(7.4)$ & $2(3.4)$ & $3(5.5)$ & $1(2.3)$ & $8(20.0)$ & $3(9.4)$ \\
\hline Eutrophic & $108(47.0)$ & $16(27.1)$ & $26(47.3)$ & $20(45.5)$ & $21(52.5)$ & $25(78.2)$ \\
\hline Overweight & $54(23.5)$ & $15(25.4)$ & $18(32.7)$ & $13(29.5)$ & $6(15.0)$ & $2(6.2)$ \\
\hline Obese & 48 (20.9) & $26(44.1)$ & $6(10.9)$ & $9(20.5)$ & $5(12.5)$ & $2(6.2)$ \\
\hline No information & $3(1.3)$ & $0(0.0)$ & $2(3.64)$ & $1(2.3)$ & $0(0.0)$ & $0(0.0)$ \\
\hline \multicolumn{7}{|l|}{ Other type of treatment prior } \\
\hline \multicolumn{7}{|l|}{ to $\mathrm{CT}$} \\
\hline Yes & $114(49.6)$ & $26(44.1)$ & $13(23.6)$ & $27(61.4)$ & $25(62.5)$ & $23(71.9)$ \\
\hline No & $115(50.0)$ & $32(54.2)$ & $42(76.4)$ & $17(38.6)$ & $15(37.5)$ & $9(28.1)$ \\
\hline No information & $1(0.4)$ & $1(1.7)$ & $0(0.0)$ & $0(0.0)$ & $0(0.0)$ & $0(0.0)$ \\
\hline \multicolumn{7}{|l|}{$\begin{array}{l}\text { Interval between diagnosis and } \\
\text { first treatment }\end{array}$} \\
\hline Mean (SD) & $50.6(44.8)$ & $48.5(41.0)$ & $41.5(58.0)$ & $63.7(38.3)$ & $49.6(34.1)$ & $54.1(41.8)$ \\
\hline Median (min-max) & $48(0-287)$ & $47(0-145)$ & $17(0-287)$ & $63(0-139)$ & $49(0-151)$ & $46(0-166)$ \\
\hline Up to 60 & $137(59.6)$ & $38(64.4)$ & $37(67.3)$ & $19(43.2)$ & $24(60.0)$ & $19(59.4)$ \\
\hline More than 60 & $75(32.6)$ & $18(30.5)$ & $16(29.1)$ & $22(50.0)$ & $11(27.5)$ & $8(25.0)$ \\
\hline No information & $18(7.8)$ & $3(5.1)$ & $2(3.64)$ & $3(6.8)$ & $5(12.5)$ & $5(15.6)$ \\
\hline \multicolumn{7}{|l|}{$\begin{array}{l}\text { Distance from residence city to } \\
\text { place of treatment }(\mathrm{km})\end{array}$} \\
\hline Mean (SD) & $95.9(145.6)$ & $83.2(115.1)$ & $81.6(111.7)$ & $117.0(178.5)$ & $92.7(158.1)$ & $119.0(180.5)$ \\
\hline Median (min-max) & $31(0-721)$ & $29(0-548)$ & $31(0-652)$ & $31(0-721)$ & $24.5(0-652)$ & $35.5(0-678)$ \\
\hline Lives in Belo Horizonte & $79(34.3)$ & $22(37.3)$ & $20(36.4)$ & $14(31.8)$ & $14(35.0)$ & $9(28.1)$ \\
\hline Up 50 & $58(25.2)$ & $13(22.0)$ & $10(18.2)$ & $12(27.3)$ & $12(30.0)$ & $11(34.4)$ \\
\hline $51-100$ & $20(8.7)$ & $5(8.5)$ & $5(9.1)$ & $3(6.8)$ & $4(10.0)$ & $3(9.4)$ \\
\hline$>100$ & $73(31.7)$ & $19(32.2)$ & $20(36.4)$ & $15(34.1)$ & $10(25.0)$ & $9(28.1)$ \\
\hline \multicolumn{7}{|l|}{ Has private health insurance (\%) } \\
\hline No & $182(79.1)$ & 47 (79.7) & $43(78.2)$ & $35(79.5)$ & $32(80.0)$ & $25(78.1)$ \\
\hline \multicolumn{7}{|l|}{ Payment of treatment (\%) } \\
\hline SUS & $214(93.0)$ & $56(94.9)$ & $49(89.2)$ & $42(95.4)$ & $38(95.0)$ & $29(90.6)$ \\
\hline Private health insurance & $10(4.3)$ & $1(1.7)$ & $3(5.5)$ & $1(2.3)$ & $2(5.0)$ & $3(9.4)$ \\
\hline Own payment/family member & $3(1.3)$ & $0(0.0)$ & $3(5.5)$ & $0(0.0)$ & $0(0.0)$ & $0(0.0)$ \\
\hline No information & $3(1.3)$ & $2(3.4)$ & $0(0.0)$ & $1(2.3)$ & $0(0.0)$ & $0(0.0)$ \\
\hline
\end{tabular}

BMI: body mass index; min-max: minimum and maximum; SD: standard deviation; SUS: Brazilian Unified National Health System. 
Table 2

Distribution of scales scores of the European Organization for Research and Treatment of Cancer Quality of Life Questionnaire Core 30 (EORTC QLQ-C30) at timepoint 1 and timepoint 2.

\begin{tabular}{|c|c|c|c|c|c|c|}
\hline \multirow[t]{2}{*}{ EORTC QLQ-C30 } & \multicolumn{2}{|c|}{$\begin{array}{c}\text { First day of the 1st cycle } \\
\text { (timepoint 1) }\end{array}$} & \multicolumn{2}{|c|}{$\begin{array}{l}\text { First day of the } 2^{\text {nd }} \text { cycle } \\
\text { (timepoint 2) }\end{array}$} & \multirow[t]{2}{*}{ z-value } & \multirow{2}{*}{$\begin{array}{c}\text { Wilcoxon paired } \\
\text { p-value }\end{array}$} \\
\hline & Mean (SD) & Median (IQR) & Mean (SD) & Median (IQR) & & \\
\hline Physical function & $80.6(23.2)$ & $93.3(33.3)$ & $80.3(23.7)$ & 86.7 (33.3) & 0.35 & 0.723 \\
\hline Role function & $81.7(28.2)$ & $100.0(33.3)$ & 78.5 (31.5) & $100.0(33.3)$ & 1.77 & 0.077 \\
\hline Emotional function & 70.6 (28.7) & $75.0(33.3)$ & 76.7 (26.3) & $83.3(33.3)$ & -3.77 & $<0.001$ * \\
\hline Cognitive function & $82.3(26.4)$ & $100.0(33.3)$ & $84.1(24.4)$ & $100.0(16.7)$ & -1.05 & 0.291 \\
\hline Social function & $80.3(25.4)$ & $100.0(33.3)$ & 76.5 (30.2) & $83.3(33.3)$ & 1.8 & 0.072 \\
\hline Fatigue & $19.6(26.3)$ & $11.1(33.3)$ & $21.1(26.8)$ & $11.1(33.3)$ & -0.74 & 0.457 \\
\hline Nausea/Vomiting & $3.3(9.1)$ & $0.0(0.0)$ & 12.5 (21.9) & $0.0(16.7)$ & -5.94 & $<0.001 *$ \\
\hline Pain & $26.2(32.1)$ & $16.7(50.0)$ & 21.9 (30.9) & $0.0(33.3)$ & 2.23 & 0.026 * \\
\hline Dyspnea & $12.2(29.2)$ & $0.0(0.0)$ & $10.8(25.6)$ & $0.0(0.0)$ & 0.88 & 0.379 \\
\hline Insomnia & $27.8(38.1)$ & $0.0(66.7)$ & $26.1(36.3)$ & $0.0(66.7)$ & 0.91 & 0.364 \\
\hline Appetite loss & $21.2(33.8)$ & $0.0(33.3)$ & 25.9 (37.9) & $0.0(66.7)$ & -1.78 & 0.076 \\
\hline Constipation & $17.8(32.2)$ & $0.0(33.3)$ & $21.5(35.8)$ & $0.0(33.3)$ & -1.44 & 0.152 \\
\hline Diarrhea & $4.2(14.5)$ & $0.0(0.0)$ & $8.0(21.1)$ & $0.0(0.0)$ & -2.37 & 0.018 * \\
\hline Financial difficulties & $26.7(36.4)$ & $0.0(33.3)$ & $28.4(35.7)$ & $0.0(66.7)$ & -0.5 & 0.617 \\
\hline $\begin{array}{l}\text { Global health status/quality } \\
\text { of life }\end{array}$ & $79.1(19.2)$ & $83.3(33.3)$ & $80.8(19.1)$ & $83.3(33.3)$ & -1.3 & 0.192 \\
\hline
\end{tabular}

IQR: interquartile; SD: standard deviation.

$\star p<0.05$.

\section{Discussion}

This study analyzed the quality of life of cancer patients with different types of cancer undergoing CT in hospitals in Belo Horizonte. We observed that in the functional quality of life scales, only the emotional domain showed a statistically significant improvement when comparing both treatment cycles. Significant changes in some symptom scales were identified. Pain, nausea/vomiting and diarrhea worsened after starting treatment. Regarding the global health status/quality of life, after starting treatment, patients with cervical cancer reported worsening, as well as patients with low weight. Widow/widower reported improvement in global health status/quality of life at timepoint 2.

In this and other studies 17,18, a significant increase in emotional function scores were identified after beginning chemotherapy treatment. The improvement can be related to the anxiety experienced by patients while waiting for CT treatment initiation. After acceptance of the diagnosis, treatment is initiated aiming disease cure, which increases patients' level of confidence. Also, treatment helps patients regain hope of improvement 19. Cancer is a stigmatizing disease, which is associated with the possibility of suffering and death. Finally, getting treated may bring comfort, improving patients' emotional status 20 .

Chemotherapeutic agents act at different stages of the cell cycle to inhibit mitosis and induce cell death. Due to their non-specific action, these drugs also affect healthy cells in the body, which explains the need for intervals between CT treatments to give cells time to recover 21 . The adverse effects of CT are primarily explained by the non-specific action of chemotherapeutic agents, and some of the most frequent are nausea and vomiting 22 . Chemotherapy-induced nausea and vomiting are common adverse effects in patients undergoing CT, and this may discourage adherence to treatment, as well as negatively affect quality of life and clinical status 23,24 .

Chemotherapeutic agents are classified according to their potential to cause nausea and vomiting, in order to define guidelines for antiemetic management. This classification is divided into high, 


\section{Table 3}

Univariate and multivariate linear regression models for global health status/quality of life determinants. Confidence intervals (CI) and p-values were calculated using the bootstrap method (2,000 simulations).

\begin{tabular}{|c|c|c|c|c|c|c|}
\hline & \multicolumn{3}{|c|}{ Univariate $(n=230)$} & \multicolumn{3}{|c|}{ Multivariate * $(n=230)$} \\
\hline & \multirow[b]{2}{*}{$\beta$ coefficient } & \multirow[b]{2}{*}{$80 \% \mathrm{Cl}$} & \multirow[b]{2}{*}{ p-value } & \multicolumn{3}{|c|}{ Final model } \\
\hline & & & & $\beta$ coefficient & $95 \% \mathrm{Cl}$ & p-value \\
\hline Intercept & & & & 44.30 & & \\
\hline Age (years) & 0.20 & $0.045 ; 0.324$ & $0.076 * *$ & & & \\
\hline \multicolumn{7}{|l|}{ Marital status } \\
\hline Married & Reference & - & - & Reference & & \\
\hline Single & -1.00 & $-5.806 ; 3.763$ & 0.807 & -0.50 & $-7.043 ; 5.978$ & 0.904 \\
\hline Divorced/separated & -2.00 & $-6.707 ; 2.364$ & 0.589 & 1.10 & $-5.429 ; 7.162$ & 0.796 \\
\hline Widower & 7.30 & $2.142 ; 12.426$ & $0.075 * *$ & 6.90 & $0.662 ; 13.153$ & $0.028 * * *$ \\
\hline \multicolumn{7}{|l|}{ Schooling (years) } \\
\hline$>12$ years & Reference & - & - & & & \\
\hline Illiterate & 3.00 & $-6.909 ; 13.290$ & 0.721 & & & \\
\hline $1-4$ & 9.30 & $1.737 ; 16.645$ & $0.116 * *$ & & & \\
\hline $5-8$ & 3.60 & $-4.060 ; 11.397$ & 0.584 & & & \\
\hline $9-12$ & 6.10 & $-1.876 ; 14.292$ & 0.341 & & & \\
\hline \multicolumn{7}{|l|}{ Types of cancer } \\
\hline Breast & Reference & - & - & Reference & & \\
\hline Colon e rectum & -0.10 & $-4.186 ; 3.624$ & 0.949 & 0.03 & $-5.364 ; 5.76$ & 0.978 \\
\hline Cervix & -7.70 & $-13.357 ;-2.207$ & $0.066 * *$ & -7.97 & $-15.289 ;-0.618$ & $0.034 * * *$ \\
\hline Head and neck & -6.50 & $-11.664 ;-1.630$ & $0.079 * *$ & -7.43 & $-15.838 ; 0.993$ & 0.074 \\
\hline Lung & -1.60 & $-6.092 ; 2.554$ & 0.595 & -4.34 & $-13.719 ; 4.585$ & 0.312 \\
\hline \multicolumn{7}{|l|}{ Comorbidity } \\
\hline None & Reference & - & - & & & \\
\hline 1 & 9.60 & $5.579 ; 13.520$ & $0.001 * *$ & & & \\
\hline 2 & 7.60 & $2.993 ; 12.348$ & $0.042 * *$ & & & \\
\hline 3 or more & 2.50 & $-2.674 ; 7.798$ & 0.567 & & & \\
\hline \multicolumn{7}{|l|}{ BMI } \\
\hline Eutrophic & Reference & - & - & Reference & & \\
\hline Low weight & -8.00 & $-15.770 ;-0.841$ & $0.150 * *$ & -9.03 & $-18.657 ;-0.823$ & $0.033 * * *$ \\
\hline Overweight & -2.70 & $-6.984 ; 1.389$ & 0.415 & -2.21 & $-8.668 ; 0.465$ & 0.465 \\
\hline Obese & 0.90 & $-3.081 ; 5.001$ & 0.746 & 0.70 & $-5.018 ; 6.325$ & 0.803 \\
\hline \multicolumn{7}{|c|}{ Other type of treatment prior to $\mathrm{CT}$} \\
\hline Yes & Reference & - & - & & & \\
\hline No & 4.50 & $1.327 ; 7.898$ & $0.070 * *$ & & & \\
\hline
\end{tabular}

BMI: body mass index; CT: chemotherapy.

Source: research data.

moderate, low, and minimal emetic potential. The two most frequent protocols in this study are classified as highly likely to cause nausea and vomiting 25 . Despite improvements over the years in different countries, guidelines for antiemetic management still show very poor adherence levels 26 . In Brazil, the SUS offers different classes of antiemetic drugs, free of cost, such as serotonin receptor antagonists (ondansetron), dopamine antagonists (metoclopramide), among others 27. Ondansetron is an antiemetic agent effective for patients undergoing treatment with high and medium-emeticpotential chemotherapeutic drugs 28 , although no protocols and therapeutic guidelines are available in Brazil for the prevention of chemotherapy-induced nausea and vomiting. Similarly to this study, we have found other studies that detected an increase in nausea and vomiting in patients undergoing CT 17,29 . 
Diarrhea also increased significantly after chemotherapy treatment. CT-induced diarrhea can occur due to changes in intestinal absorption caused by chemotherapeutic agents, in addition to biochemical and microbial changes 30 . Other studies have also reported increased diarrhea after CT 11,13,31.

A systematic review 32 showed that CT improves pain control, supporting our findings indicating that pain considerably decreased after CT. The main targets of CT are: control of the disease, mass shrinking, cure, and palliative care. Despite the growing number of studies on pain, the better understanding of its mechanisms and the increase in the consumption of drugs to relieve the suffering, it was found in another systematic review that the prevalence of pain among cancer patients remains high. A possible explanation for this is the change in the profile of cancer patients, which are living longer and are more likely to report their pain 33. Pain may be caused by invasion or compression of adjacent structures by the tumor and may be related to adverse effects of cancer treatments 34 . Identifying pain in cancer patients and treating it is extremely important due to the impact on their quality of life 35 .

In this study, widowhood was a predictor of changes in the global health status/quality of life after beginning CT, when compared to married subjects. Older age is associated with widowed status, and older adults experience fewer negative emotions than younger people. Besides, when these emotions are experienced, they are also not as intense (for the older adults) 36 . As time passes and people age, they realize time is finite and gain a deeper appreciation for emotional meanings 37 , which may explain the finding that a widowed status was associated with improvements in the global health status/quality of life after CT. Another possible explanation is the attention received from healthcare professionals during disease. Studies have shown that widowed subjects report feeling more lonely than married subjects 38,39 , therefore contact with the team involved in their care may be related to a positive self-perception of quality of life in this group of patients.

The type of cancer was also a predictor of changes in the global health status/quality of life after beginning the CT treatment. The presence of cervical cancer is associated with quality of life deterioration after chemotherapy, compared to breast cancer. The type of treatment for cervical cancer depends on the stage of the disease, and also on the clinical conditions of the patient. Most women treated with CT are also undergoing concomitant radiation therapy 40 . Several factors can affect the quality of life in women undergoing treatment for cervical cancer, including changes in sexual functioning due to vaginal stenosis, decreased libido, and pain during intercourse, in addition to symptoms such as lymphedema and urinary and fecal incontinence 41 . A relevant point to be discussed is the relationship between this type of cancer and poverty 42 . In this study, a significant difference in individual income and years of schooling was found between women with breast cancer and cervical cancer, with lower means in the latter group. Some studies have found a negative association between decreased quality of life and low income among cancer patients 43,44,45.

Body mass index (BMI) is an international indicator of the nutritional status of individuals. The cutoff points used for this calculation follow the recommendation of the WHO, namely: $<18.5 \mathrm{~kg} /$ $\mathrm{m}^{2}$ (underweight), $>18.5 \mathrm{~kg} / \mathrm{m}^{2}$ and $<25 \mathrm{~kg} / \mathrm{m}^{2}$ (ideal weight range), $>25 \mathrm{~kg} / \mathrm{m}^{2}$ and $<30 \mathrm{~kg} / \mathrm{m}^{2}$ (overweight) and $>30 \mathrm{~kg} / \mathrm{m}^{2}$ (obese) 46 . Cancer-associated cachexia is a multifactorial disorder, characterized mainly by loss of body weight resulting not only from adipose tissue but also from muscle mass. There is still no medical intervention capable of reversing the nutritional support of patients affected by cachexia, and this disorder compromises patients' physical and emotional functions as well as their quality of life 47 . One of the classifications for cachexia is a BMI $<20 \mathrm{~kg} / \mathrm{m}^{2} 48$. This syndrome is associated with deterioration of patient's quality of life 49,50, confirming the findings of this study, in which underweight patients reported poorer global health status/quality of life after the onset of treatment, when compared to those in their normal weight range. One explanation for this deteriorated quality of life is the increased toxicity inherent to the treatment, due to loss of muscle 47.

Similar to another study 51, it draws attention to the large number of patients who did not start treatment within 60 days, as advocated by Brazilian regulation 52 that guarantees the right of patients with malignant neoplasia to begin their first treatment in SUS within a maximum period of 60 days after diagnosis. Although this study did not find an association of changes in the quality of lifeL of patients with the interval between diagnosis and the start of treatment, the delay in beginning treat- 
ment can negatively affect the patients' survival 53,54 and the agility to beginning treatment improves quality of life 54 .

One of the limitations of this study is the poor representativeness of some high-incidence cancers that use CT in a small portion of the population, such as prostate cancer, for example. Also, we collected data at the beginning of CT, and therefore could not assess the variability of quality of life predictors over the entire course of treatment. Another significant limitation of this study is the loss of patient data due to incomplete hospital records and absence of analysis for each type of cancer separately.

\section{Conclusion}

The adverse effects of CT identified in this study, such as nausea, vomiting, and diarrhea, have major impacts on the quality of life of cancer patients. Despite its physical deterioration consequences, we also observed that (chemo)therapy had a positive effect on patients' emotional state which may contribute and arouse future discussions. Furthermore, we observed that individual characteristics are associated with changes in the global health status/quality of life of patients after treatment initiation. Assessing the quality of life of patients with different types of cancer on CT using their clinical and individual characteristics may help identify those who are more vulnerable to quality of life deterioration and provide information to support decisions made by healthcare professionals who deal with the care of patients with different neoplasms. With the growing advances in diagnosis and treatment of malignant tumors, we are now aiming to change prevalence and provide longer survivals. Therefore, it is greatly significant to consider the change in the comfort level expected by cancer patients. Studies looking at the quality of life of patients with cancer are important to generate information for healthcare professionals to ensure patients a dignified and functional life by monitoring signs and symptoms not only inherent to the disease, but also arising from therapy and patients' individual characteristics. 


\section{Contributors}

D. P. Moreira contributed in the study conception and design, data collection, data analysis and interpretation, statistical analysis, preparation and revision of this manuscript, and assume full responsibility for the study, ensuring proper investigation and clarification of issues. G. P. R. Simino contributed in the study conception and design, data collection, data analysis and interpretation, statistical analysis, preparation and revision of this manuscript, and assume full responsibility for the study, ensuring proper investigation and clarification of issues. I. A. Reis and M. A. C. Santos contributed in the data analysis and interpretation, statistical analysis, preparation and revision of this manuscript, and assume full responsibility for the study, ensuring proper investigation and clarification of issues. M. L. Cherchiglia contributed in the study conception and design, data collection, data analysis and interpretation, statistical analysis, preparation and revision of this manuscript, and assume full responsibility for the study, ensuring proper investigation and clarification of issues regarding.

\section{Additional informations}

ORCID: Daniela Pena Moreira (0000-0002-31091342); Giovana Paula Rezende Simino (0000-00029814-3004); Ilka Afonso Reis (0000-0001-71998590); Marcos Antonio da Cunha Santos (00000002-5228-8822); Mariangela Leal Cherchiglia (0000-0001-5622-567X).

\section{Acknowledgments}

This study was funded by the Brazilian National Research Council (CNPq, grant n. 456360/20140; Minas Gerais Research Foundation (FAPEMIG, grant n. CDS - PPM-00716-15) and Brazilian Graduate Studies Coordinating Board (CAPES, finance code 001).

\section{Conflict of interest}

All authors declare no potential conflict of interest related to this article and have no other relevant financial interests to declare.

\section{References}

1. Ferlay J, Colombet M, Soerjomataram I, Mathers C, Parkin DM, Piñeros M, et al. Estimating the global cancer incidence and mortality in 2018: GLOBOCAN sources and methods. Int J Cancer 2019; 144:1941-53.

2. Instituto Nacional de Câncer José Alencar Gomes da Silva. Estimativa 2020: incidencia de câncer no Brasil. https://www.inca.gov.br/si tes/ufu.sti.inca.local/files//media/document// estimativa-2020-incidencia-de-cancer-nobrasil.pdf (accessed on 24/Aug/2020).

3. Saeedi-Saedi H, Shahidsales S, Koochak-Pour M, Sabahi E, Moridi I. Evaluation of emotional distress in breast cancer patients. Iran J Cancer Prev 2015; 8:36-41.

4. Ahles TA, Root JC. Cognitive effects of cancer and cancer treatments. Annu Rev Clin Psychol 2018; 14:425-51.

5. World Health Organization Quality of Life Group. The World Health Organization quality of life assessment (WHOQOL): position paper from the World Health Organization. Soc Sci Med 1995; 41:1403-9.

6. Seidl EMF, Zannon CMLC. Qualidade de vida e saúde: aspectos conceituais e metodológicos Cad Saúde Pública 2004; 20:580-8.

7. Kluetz PG, O'Connor DJ, Soltys K. Incorporating the patient experience into regulatory decision making in the USA, Europe, and Canada. Lancet Oncol 2018; 19:e267-74.

8. Yang LY, Manhas DS, Howard AF, Olson RA. Patient-reported outcome use in oncology: a systematic review of the impact on patientclinician communication. Supportive Care in Cancer 2018; 26:41-60.

9. Velikova G, Booth L, Smith AB, Brown PM, Lynch P, Brown JM, et al. Measuring quality of life in routine oncology practice improves communication and patient well-being: a randomized controlled trial. J Clin Oncol 2004; 22:714-24

10. Velikova G, Keding A, Harley C, Cocks K, Booth L, Smith AB, et al. Patients report improvements in continuity of care when quality of life assessments are used routinely in oncology practice: Secondary outcomes of a randomised controlled trial. Eur J Cancer 2010; 46:2381-8

11. Büttner M, Zebralla V, Dietz A, Singer S. Quality of life measurements: any value for clinical practice? Curr Treat Options Oncol 2017; 18:30.

12. Minayo MCS, Hartz ZMA, Buss PM. Quality of life and health: a necessary debate. Ciênc Saúde Colet 2000; 5:7-18.

13. Carlotto A, Hogsett VL, Maiorini EM, Razulis JG, Sonis ST. The economic burden of toxicities associated with cancer treatment: review of the literature and analysis of nausea and vomiting, diarrhoea, oral mucositis and fatigue. Pharmacoeconomics 2013; 31:753-66. 
14. Simino GPR, Reis IA, Acurcio FA, Andrade EIG, Brazil NML, Cherchiglia ML. Fatores de risco associados a náuseas e vômitos induzidos por quimioterapia antineoplásica. Rev Saúde Pública 2020; 54:106.

15. Aaronson NK, Ahmedzai S, Bergman B, Bullinger M, Cull A, Duez NJ, et al. The European Organization for Research and Treatment of Cancer QLQ-C30: a quality-of-life instrument for use in international clinical trials in oncology. J Natl Cancer Inst 1993; 85:365-76.

16. Fayers PM, Aaronson NK, Bjordal K, Groenvold $\mathrm{M}$, Curran D, Bottomley A. EORTC QLQ-C30 scoring manual. 3rd Ed. https:// www.eortc.org/app/uploads/sites/2/2018/02/ SCmanual.pdf (accessed on 29/Aug/2020).

17. Lofrano AD, Coura CPM SM. Avaliação da qualidade de vida de mulheres com carcinoma do colo do útero em quimioterapia paliativa. Rev Bras Cancerol 2016; 62:203-13.

18. Kramer JA, Curran D, Piccart M, De Haes JCJM, Bruning PF, Klijn JGM, et al. Randomised trial of paclitaxel versus doxorubicin as first-line chemotherapy for advanced breast cancer: quality of life evaluation using the EORTC QLQ-C30 and the Rotterdam Symptom Checklist. Eur J Cancer 2000; 36:1488-97.

19. Villar RR, Fernández SP, Garea CC, Pillado MTS, Barreiro VB, Martín CG. Quality of life and anxiety in women with breast cancer before and after treatment. Rev Latinoam Enferm (Online) 2017; 25:e2958.

20. Stergiou-Kita M, Pritlove C, Kirsh B. The "Big C” - stigma, cancer, and workplace discrimination. J Cancer Surviv 2016; 10:1035-50.

21. Bruton LL, Lazo JS, Parker KL. Goodman \& Gilman's. The pharmacological basis of therapeutics. 12th Ed. New York: McGraw-Hill; 2011.

22. Bonassa EM, Gato MI. Terapêutica oncológica para enfermeiros e farmacêuticos. Terapêutica oncológica para enfermeiros e farmacêuticos. 4th Ed. Rio de Janeiro: Atheneu; 2012.

23. Rapoport BL. Delayed chemotherapy-induced nausea and vomiting: pathogenesis, incidence, and current management. Front Pharmacol 2017; 8:19.

24. Yokoe T, Hayashida T, Nagayama A, Nakashoji A, Maeda H, Seki T, et al. Effectiveness of antiemetic regimens for highly emetogenic chemotherapy-induced nausea and vomiting: a systematic review and network meta-analysis. Oncologist 2019; 24:e347-57.

25. Razvi Y, Chan S, McFarlane T, McKenzie E, Zaki P, DeAngelis C, et al. ASCO, NCCN, MASCC/ESMO: a comparison of antiemetic guidelines for the treatment of chemotherapyinduced nausea and vomiting in adult patients. Support Care Cancer 2019; 27:87-95.

26. Navari RM, Aapro M. Antiemetic prophylaxis for chemotherapy-induced nausea and vomiting. N Engl J Med 2016; 374:1356-67.
27. Ministério da Saúde. Relação Nacional de Medicamentos Essenciais 2020. http://bvsms. saude.gov.br/bvs/publicacoes/relacao_medi camentos_rename_2020.pdf (accessed on 24/ Aug/2020).

28. Simino GPR, Marra LP, Andrade EIG, Acúrcio FA, Reis IA, Araújo VE, et al. Efficacy, safety and effectiveness of ondansetron compared to other serotonin-3 receptor antagonists (5-HT3RAs) used to control chemotherapy-induced nausea and vomiting: systematic review and meta-analysis. Expert Rev Clin Pharmacol 2016; 9:1183-94.

29. Klafke N, Mahler C, von Hagens C, Uhlmann L, Bentner M, Schneeweiss A, et al. The effects of an integrated supportive care intervention on quality of life outcomes in outpatients with breast and gynecologic cancer undergoing chemotherapy: results from a randomized controlled trial. Cancer Med 2019; 8:3666-76.

30. Stein A, Voigt W, Jordan K. Review: chemotherapy-induced diarrhea: pathophysiology, frequency and guideline-based management. Ther Adv Med Oncol 2010; 2:51-63.

31. Machado SM, Sawada NO. Evaluation quality of life in oncology patients submitted to adjuvant. Texto Context Enferm 2008; 17:750-7.

32. Kristensen A, Vagnildhaug OM, Grønberg BH, Kaasa S, Laird B, Solheim TS. Does chemotherapy improve health-related quality of life in advanced pancreatic cancer? A systematic review. Crit Rev Oncol Hematol 2016; 99:286-98.

33. Van Den Beuken-Van Everdingen MHJ, Hochstenbach LMJ, Joosten EAJ, Tjan-Heijnen VCG, Janssen DJA. Update on prevalence of pain in patients with cancer: systematic review and meta-analysis. J Pain Symptom Manage 2016; 51:1070-90.

34. Money S, Garber B. Management of cancer pain. Curr Emerg Hosp Med Rep 2018; 6:141-6.

35. Puetzler J, Feldmann RE, Brascher AK, Gerhardt A, Benrath J. Improvements in healthrelated quality of life by comprehensive cancer pain therapy: a pilot study with breast cancer outpatients under palliative chemotherapy. Oncol Res Treat 2014; 37:456-62.

36. English T, Carstensen LL. Selective narrowing of social networks across adulthood is associated with improved emotional experience in daily life. Int J Behav Dev 2014; 38:195-202.

37. Carstensen LL. The influence of a sense of time on human development. Science 2006; 312:1913-5.

38. Azeredo ZAS, Afonso MAN. Solidão na perspectiva do idoso. Rev Bras Geriatr Gerontol 2016; 19:313-24.

39. Grover S, Avasthi A, Sahoo S, Lakdawala B, Dan A, Nebhinani N, et al. Relationship of loneliness and social connectedness with depression in elderly: a multicentric study under the aegis of Indian Association for Geriatric Mental Health. J Geriatr Ment Heal 2018; 5:99-106. 
40. Santos M, Corrêa TS, Faria LDB, Siqueira GSM, Reis PED, Pinheiro RN. Diretrizes oncológicas 2. São Paulo: Doctor Press Ed. Científica; 2019.

41. Cohen PA, Jhingran A, Oaknin A, Denny L. Cervical cancer. Lancet 2019; 393:169-82.

42. Ginsburg O, Bray F, Coleman MP, Vanderpuye V, Eniu A, Kotha SR, et al. The global burden of women's cancers: a grand challenge in global health. Lancet 2017; 389:847-60.

43. Ell K, Xie B, Wells A, Nedjat-Haiem F, Lee PJ, Vourlekis B. Economic stress among low-income women with cancer: effects on quality of life. Cancer 2008; 112:616-25.

44. Fenn KM, Evans SB, McCorkle R, DiGiovanna MP, Pusztai L, Sanft T, et al. Impact of financial burden of cancer on survivors' quality of life. J Oncol Pract 2014; 10:332-8.

45. Lathan CS, Cronin A, Tucker-Seeley R, Zafar SY, Ayanian JZ, Schrag D. Association of financial strain with symptom burden and quality of life for patients with lung or colorectal cancer. J Clin Oncol 2016; 34:1732-40.

46. World Health Organization. Waist circumference and waist-hip ratio: report of a WHO Expert Consultation. Geneva: World Health Organization; 2008.

47. Baracos VE, Martin L, Korc M, Guttridge DC, Fearon KCH. Cancer-associated cachexia. Nat Rev Dis Primers 2018; 4:17105.

48. Fearon K, Strasser F, Anker SD, Bosaeus I, Bruera E, Fainsinger RL, et al. Definition and classification of cancer cachexia: An international consensus. Lancet Oncol 2011; 12:489-95.
49. Takayama K, Atagi S, Imamura F, Tanaka H, Minato K, Harada T, et al. Quality of life and survival survey of cancer cachexia in advanced non-small cell lung cancer patients - Japan nutrition and QOL survey in patients with advanced non-small cell lung cancer study. Support Care Cancer 2016; 24:3473-80.

50. Crawford J. Cancer cachexia: are we ready to take a step forward? Cancer 2018; 124:456-8.

51. Medeiros GC, Bergmann A, Aguiar SS, Thuler LCS. Análise dos determinantes que influenciam o tempo para o início do tratamento de mulheres com câncer de mama no Brasil. Cad Saúde Pública 2015; 31:1269-82.

52. Brasil. Lei no 12.732 , de 22 de novembro de 2012. Dispõe sobre o primeiro tratamento de paciente com neoplasia maligna comprovada e estabelece prazo para seu início. Diário Oficial da União 2012; 23 nov.

53. Corrêa CS, Lima AD, Leite IC, Pereira LC, Nogueira MC, Duarte DD, et al. Rastreamento do câncer do colo do útero em Minas Gerais: avaliação a partir de dados do Sistema de Informação do Câncer do Colo do Útero (SISCOLO). Cad Saúde Colet (Rio J.) 2017; 25:315-23.

54. Neal RD, Tharmanathan P, France B, Din NU, Cotton S, Fallon-Ferguson J, et al. Is increased time to diagnosis and treatment in symptomatic cancer associated with poorer outcomes? Systematic review. Br J Cancer 2015; 112 Suppl 1:S92-107. 


\section{Resumo}

O estudo teve como objetivo avaliar as mudanças na qualidade de vida de pacientes oncológicos no início do primeiro e segundo ciclos de quimioterapia (QT) em hospitais em Belo Horizonte, Minas Gerais, Brasil. Foi realizado um estudo descritivo, prospectivo e longitudinal com uma abordagem quantitativa. Arrolamos 230 pacientes de uma coorte maior, diagnosticados com os cinco tipos de câncer mais frequentes (mama, colorretal, colo uterino, pulmão e cabeça e pescoço), com idade 18 anos ou mais e que estavam no início da QT. A qualidade de vida foi avaliada com o EORTC QLQ-C30, versão 3, aplicado no início do primeiro e segundo ciclos de QT. O teste pareado de Wilcoxon foi utilizado para identificar diferenças na qualidade de vida entre os dois momentos. Para investigar potenciais preditores de estado de saúde global/qualidade de vida, foi realizada uma análise de regressão linear multivariada com o método bootstrap. Houve um aumento significativo na pontuação da função emocional dos pacientes $(p<0,001)$ e de dor $(p=0,026)$, diarreia ( $p$ $=0,018)$ e náusea/vômito $(p<0,001)$ após o início da quimioterapia. Estado civil "viúvo/a" esteve associado a melhoras no estado de saúde global/qualidade de vida $(p=0,028)$, enquanto presença de câncer do colo uterino $(p=0,034)$ e baixo peso $(p=$ $0,033)$ estiveram relacionados a piores resultados no estado de saúde global/qualidade de vida. A QT tem efeitos deletérios na saúde física dos pacientes, mas leva a melhorias no domínio emocional. As características individuais dos pacientes no início da QT estão associadas a mudanças na qualidade vida. Nosso estudo pode ajudar a identificar essas características.

Neoplasias; Qualidade de Vida; Tratamento Farmacológico; Perfil de Saúde; Citotoxinas

\section{Resumen}

El objetivo del presente estudio fue evaluar cambios en la calidad de vida de pacientes con cáncer, entre el principio del primero y segundo ciclo de quimioterapia (CT), en hospitales en Belo Horizonte, Brasil. Se llevó a cabo un estudio longitudinal, prospectivo, descriptivo con un enfoque cuantitativo. Participaron 230 pacientes, de una cohorte más amplia, diagnosticados con los cinco tipos de cáncer más frecuentes (pecho, colorrectal, cervical, pulmón, cabeza y cuello), con 18 años y más, que estaban comenzando CT por primera vez. La calidad de vida fue evaluada mediante EORTC QLQ-C30 versión 3, aplicada al comienzo del primer y segundo ciclo de quimioterapia. Se usó el test pareado de Wilcoxon para identificar las diferencias en calidad de vida entre dos puntos en el tiempo. Para investigar los predictores potenciales del estatus de salud global/calidad de vida, se realizó un análisis lineal multivariado, usando el método de Bootstrap. Hubo un aumento significativo en las puntuaciones de las funciones emocionales de los pacientes $(p<0,001)$, así como las puntuaciones para dolor $(p=0,026)$, diarrea $(p=0,018)$ y náusea/vómitos $(p<0,001)$ tras el comienzo de la quimioterapia. Ser viudo/a estuvo asociado con mejoras en el estatus de salud global/ calidad de vida $(p=0,028)$, mientras que la presencia de cáncer cervical $(p=0,034)$ y estar por debajo del peso $(p=0,033)$ estuvieron relacionados con puntuaciones más bajas estatus de salud global/calidad de vida. La CT tiene efectos perjudiciales en la salud física de los pacientes, sin embargo, por otro lado, conduce a mejoras en el ámbito emocional. Las características individuales de pacientes al comienzo de la CT están asociadas con cambios en su calidad de vida. Nuestro estudio podría ayudar a identificar estas características.

Neoplasias; Calidad de Vida; Quimioterapia;

Perfil de Salud; Citotoxinas
Recebido em 07/Jan/2020

Versão final reapresentada em 12/Nov/2020

Aprovado em 23/Nov/2020 